\title{
A Retrospective Cross-Sectional Study of Severe Breakthrough SARS-CoV-2 Infection in the General Population Requiring Hospitalization Within a Single Health System
}

\author{
Roshan Acharya ${ }^{\text {a, d }}$ (D), Smita Kafle ${ }^{\text {b }}$, Natalie Kandinata ${ }^{a}$, Brian Slipman ${ }^{a}$, \\ Meera Ghimire ${ }^{a}$, Andrew B. Trotter ${ }^{\mathrm{c}}$
}

\begin{abstract}
Background: Despite coronavirus disease 2019 (COVID-19) vaccination efforts, severe acute respiratory syndrome coronavirus 2 (SARS-CoV-2) infections in vaccinated individuals ("breakthrough SARS-CoV-2 infections") have emerged. Our understanding of breakthrough SARS-CoV-2 infections continues to evolve, and there is a paucity of information describing severe breakthrough SARSCoV-2 infections. We conducted this study with the aim of describing breakthrough SARS-CoV-2 infections requiring hospitalization and exploring factors associated with severe breakthrough infection.
\end{abstract}

Methods: The study included patients within our health network who received at least one dose of a messenger RNA (mRNA) COVID-19 vaccine and required hospitalization due to breakthrough SARSCoV-2 infection from January 1 to August 15, 2021. We performed a descriptive analysis of vaccinated patients requiring hospitalization. Multivariable logistic regression (LR) analysis was performed to explore factors associated with severe breakthrough infection.

Results: Out of 67,223 vaccinated individuals, 78 (0.12\%) patients were hospitalized with breakthrough SARS-CoV-2 infection, of which 25 individuals $(0.04 \%$ of those vaccinated, and $32 \%$ of all hospitalized) developed severe infection. The mean age of those with breakthrough infection was 72 years, the majority were White $(60 \%)$, and dyspnea was the most common reason for hospital admission $(53 \%)$, with bimodal peaks of hospitalization in January-February (40\%) and July-August (34\%). In LR analysis, male patients had 4.03

Manuscript submitted January 8, 2022, accepted January 20, 2022

Published online January 29, 2022

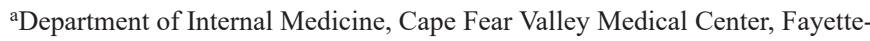
ville, NC 28304, USA

${ }^{b}$ Fayetteville State University School of Nursing, Fayetteville, NC 28301, USA 'Division of Infectious Disease, Department of Medicine, University of Illinois at Chicago, Chicago, IL 60612, USA

${ }^{\mathrm{d} C}$ Corresponding Author: Roshan Acharya, Department of Internal Medicine, Cape Fear Valley Medical Center, Fayetteville, NC 28306, USA.

Email: roshan.ach@gmail.com

doi: https://doi.org/10.14740/jocmr4662 times the odds of developing severe SARS-CoV-2 infection than female patients (adjusted odds ratio (aOR): 4.03, 95\% confidence interval (CI): 1.21 - 13.40), and an immunocompromising condition had 6.32 times the odds of developing severe COVID-19 disease (aOR: 6.32, 95\% CI: 1.48 - 26.18).

Conclusions: The rate of severe breakthrough SARS-CoV-2 infection was very low, and male sex and immunocompromising conditions were associated with severe breakthrough infection. Clinicians and health systems should continue to campaign for COVID-19 vaccination aggressively.

Keywords: Breakthrough infection; COVID-19; SARS-CoV-2; Vaccination

\section{Introduction}

Prior to the development of vaccines against severe acute respiratory syndrome coronavirus 2 (SARS-CoV-2), the coronavirus disease 2019 (COVID-19) pandemic had already claimed at least 3 million lives globally [1]. Two vaccines developed were found to have very high efficacy in human trials. The BNT162b2 vaccine developed by Pfizer-BioNTech was reported 95\% effective in preventing COVID-19 in patients greater than or equal to 16 years of age [2]. Similarly, the messenger RNA (mRNA)-1273 vaccine developed by Moderna Inc. was reported 94\% effective against COVID-19 in age 18 years and above [3]. The reported efficacy in the trial by Pfizer-BioNTech was 7 days after the second dose, whereas 14 days after the second dose in the trial by Moderna Inc. The BNT162b2 vaccine's efficacy was found to be $92 \%$ and $95 \%$ against COVID-19 in patients greater than or equal to 16 years of age in two non-clinical trials, real-world studies from Israel $[4,5]$.

Despite the development and roll-out of effective vaccines against SARS-CoV-2, the emergence of new variants of SARSCoV-2 and post-vaccination SARS-CoV-2 infection (also referred to as breakthrough infection) have been described. The BNT162b2 vaccine and mRNA-1273 vaccine reportedly had $0.05 \%$ (eight cases/18,198 vaccinated) and $0.09 \%$ (12 cas- 
es/14,550 vaccinated) breakthrough infections, respectively, during clinical trials $[2,3]$. After the roll-out of the COVID-19 vaccination, the first cases of breakthrough infections in the USA were reported in New York [6]. Since then, breakthrough infections continue to be described, and there has been an increase in reported cases of breakthrough infections [7-17]. According to the Centers for Disease Control and Prevention (CDC), 10,262 breakthrough infections were reported from 46 states and territories of the USA as of April 30, 2021, of which 995 (10\%) patients were known to be hospitalized, and 160 (2\%) patients died [18]. In general, breakthrough infections appear to be uncommon and less severe than SARS-CoV-2 infections in unvaccinated adults. However, breakthrough infections continue to be reported, and our understanding of predictors of breakthrough infection and severity continues to evolve.

Beginning in May 2021, CDC transitioned from monitoring all reported COVID-19 breakthrough infections to investigating only severe breakthrough COVID-19 cases [18]. This has resulted in limitations in available information such as demographics, baseline comorbidities, and risk factors for breakthrough infections.

We conducted this retrospective observational study to investigate the rate of breakthrough SARS-CoV-2 infections within our health network and to study the demographics, baseline comorbidities, and risk factors in breakthrough COVID-19 cases.

\section{Materials and Methods}

\section{Study design and study population}

This study was a retrospective, observational cross-sectional study of patients admitted to four hospitals in the Cape Fear Valley Health System (CFVHS), which has 950 total inpatient beds and provides care for six counties in the southeastern region of North Carolina. CFVHS started Pfizer-BioNTech mRNA vaccination of front-line health workers on December 15, 2020, and offered vaccination to the general public on January 5, 2021. The Moderna vaccine was introduced on January 26, 2021.

Patients were included in this study if they received at least one dose of an mRNA COVID-19 vaccine and were admitted to any of the CFVHS hospitals from January 1, 2021, to August 15, 2021, with the diagnosis of SARS-CoV-2 infection. International Classification of Disease, the 10th Revision (ICD-10) codes U.07.1, Z11.52 were used to identify possible eligible patients with SARS-CoV-2 infection, and a positive reverse transcription-polymerase chain reaction (RT-PCR) test using a nasopharyngeal (NP) swab was used to confirm SARSCoV-2 infection.

Baseline demographic information, comorbidities including diabetes mellitus (DM), obesity, smoking, malignancy on active chemotherapy, vital signs at presentation, and baseline laboratory testing such as complete blood cell count and comprehensive metabolic profile, were collected. Symptoms of SARS-CoV-2 infection were defined as any of the following symptoms: fever, cough, difficulty breathing, weakness and others. The category other symptoms included palpitations, dizziness, chest pressure, fall, abdominal pain and vomiting. Renal deterioration was defined as acute kidney injury (AKI) per KDIGO-2012 criteria, hepatic deterioration was defined as liver transaminases that were $\geq 5$ times the upper normal limits, and neurological deterioration was defined by a change in mental status. Patients were considered immunocompromised if patients were receiving any chronic corticosteroid therapy or immunotherapy for chronic inflammatory disease (CID), current chemotherapy for malignancy, solid organ transplant (SOT) recipients, or acquired immunodeficiency syndrome (AIDS) patients. Cluster of differentiation (CD4) count $<200$ cells $/ \mathrm{mm}^{3}$ or ever having a diagnosis of AIDS defined a patient as having AIDS in our study. For those who only received one dose of the COVID-19 vaccine (partially vaccinated) at the time of SARSCoV-2 infection, the date of the single-dose was considered day 0 . For those who received both doses of the COVID-19 vaccine, the date of their second dose was considered day 0 .

The study was approved, and the informed consent requirement was waived by the Institutional Review Board (IRB) of CFVHS (IRB ID: NR1085-21); and this study was conducted in compliance with all the applicable institutional ethical guidelines on human subjects.

\section{Inclusion criteria}

The inclusion criteria were: 1) Patients vaccinated against SARS-CoV-2 with at least one dose of BNT162b2 (PfizerBioNTech) or mRNA-1273 (Moderna) vaccine; 2) Positive SARS-CoV-2 RT-PCR test from NP swab at any time after vaccination; 3) Admitted to inpatient bed from January 1 to August 15, 2021; 4) Admitted for symptomatic SARS-CoV-2 infection; 5) Age $\geq 16$ years.

\section{Exclusion criteria}

Exclusion criteria were: 1) Patients who were not discharged from the hospital by August 15, 2021;2) Emergency department (ED) visits without hospitalization; 3) Patients who had a positive RT-PCR test but were not admitted for COVID-19 related symptoms; 4) Asymptomatic SARS-CoV-2 infection.

From December 15, 2020, to August 15, 2021, CFVHS fully vaccinated 59,095 individuals and partially vaccinated 8,128 individuals. During the same period, there were 1,542 hospitalizations with an associated COVID-19 ICD-10 diagnosis. A total of 81 patients were identified as COVID-19 hospitalizations post COVID-19 vaccination, while 1,461 patients were excluded from the study due to failure to meet the inclusion criteria of being vaccinated against SARS-CoV-2. Of 81 hospitalizations, three patients were determined to have been hospitalized for problems unrelated to SARS-CoV-2 infection during the chart review and were excluded from the study. The final sample size of the study was 78 patients. Out of 78, 39 were fully vaccinated, and 39 were partially vaccinated individuals. The patients were classified into fully vaccinated and partially vaccinated groups for comparison between the groups (Table 1). 
Table 1. Baseline Characteristics of Patients With Breakthrough SARS-CoV-2 Infections

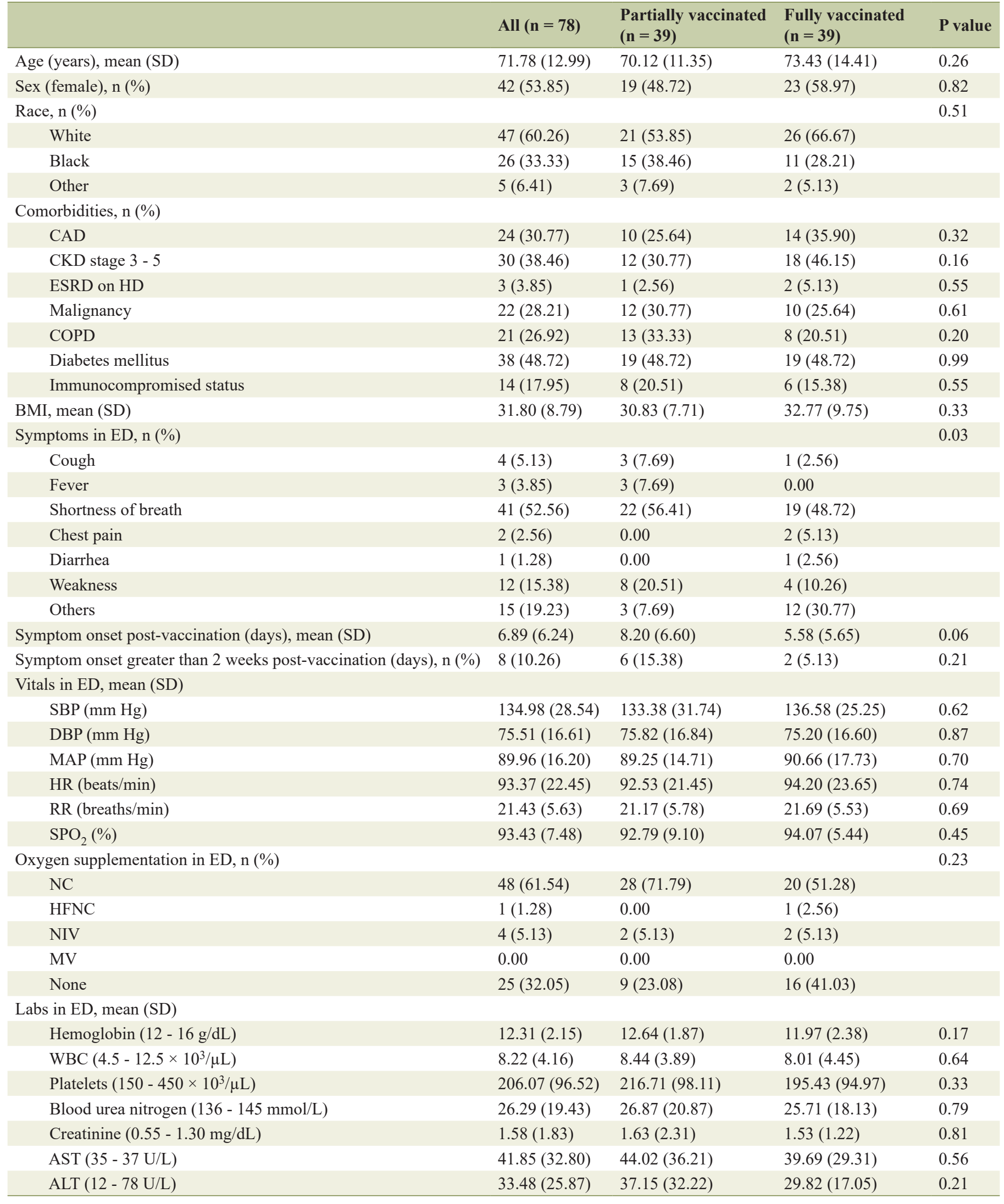


Table 1. Baseline Characteristics of Patients With Breakthrough SARS-CoV-2 Infections - (continued)

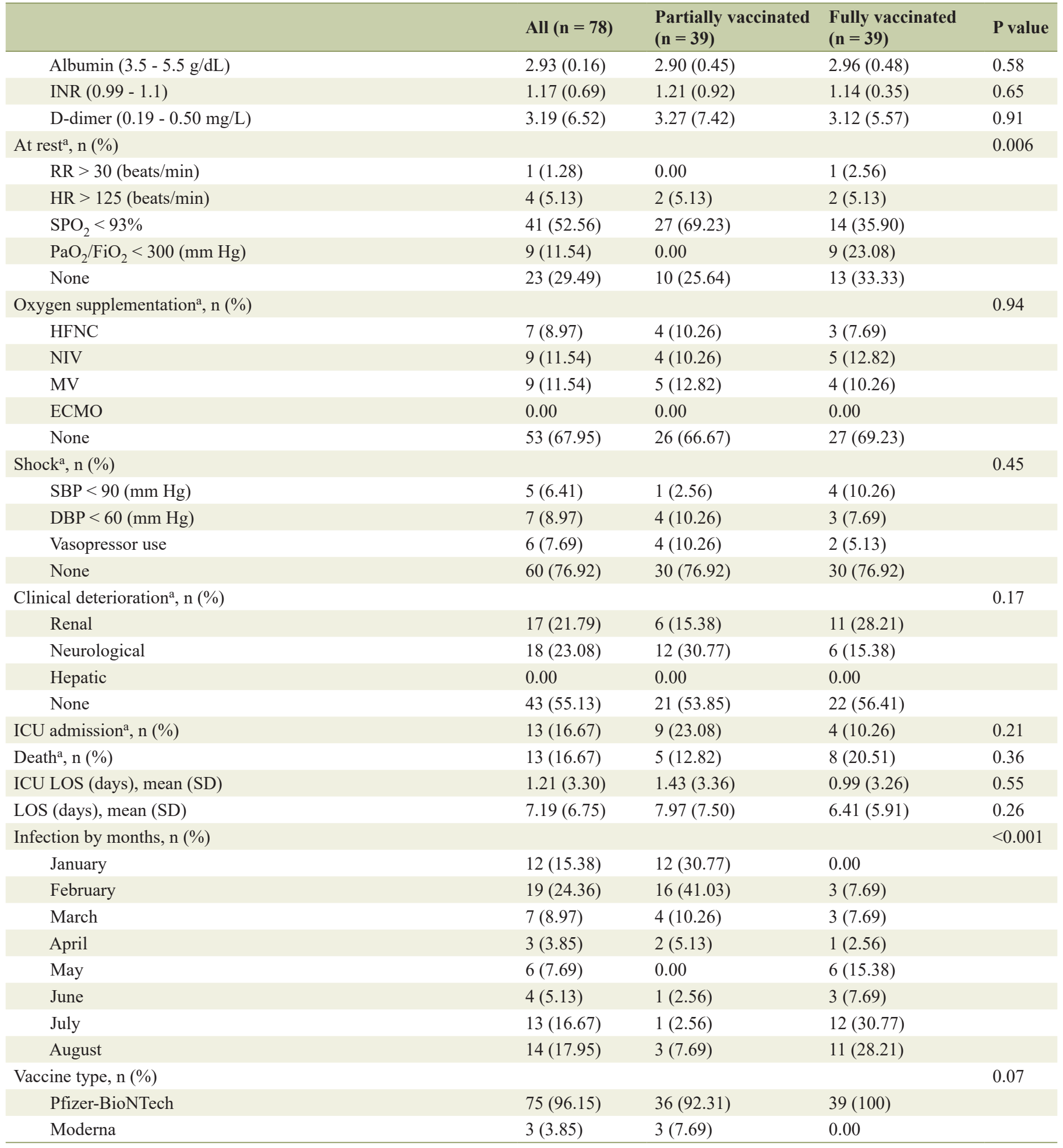

alf developed any time during the hospitalization. SARS-CoV-2: severe acute respiratory syndrome coronavirus 2; SD: standard deviations; CAD: coronary artery disease; CKD: chronic kidney disease; ESRD: end-stage renal disease; HD: hemodialysis; COPD: chronic obstructive pulmonary disease; BMI: body mass index; SBP: systolic blood pressure; DBP: diastolic blood pressure; MAP: mean arterial pressure; HR: heart rate; RR: respiratory rate; $\mathrm{SPO}_{2}$ : oxygen saturation; NC: nasal cannula; HFNC: high-flow nasal cannula; NIV: noninvasive mechanical ventilation; MV: invasive mechanical ventilation; WBC: white blood cell; AST: aspartate aminotransferase; $\mathrm{ALT}$ : alanine aminotransferase; INR: international normalized ratio; $\mathrm{PaO}_{2} / \mathrm{FiO}_{2}$ : ratio of partial pressure of oxygen to fractional inspired oxygen; ECMO: extra corporeal membrane oxygenation; ICU: intensive care unit; LOS: length of stay. 


\section{Outcomes}

\section{Primary outcome}

The primary outcome was the hospitalization in vaccinated individuals due to SARS-CoV-2 infection (breakthrough infection).

\section{Secondary outcomes}

The secondary outcomes were: 1) Severe SARS-CoV-2 infection as defined below; 2) Number of days post-vaccination COVID-19 symptoms started; 3) Length of hospital stay; 4) Hospitalization outcome (survival and discharge or death).

\section{Study definition}

The definition utilized for severe COVID-19 disease was modified from the definition used by the Pfizer-BioNTech and Moderna COVID-19 vaccine randomized controlled trials (RCTs) $[2,3]$.

Severe COVID-19 disease was defined as a positive COVID-19 RT-PCR test and at least one of the following: 1) Ratio of partial pressure of oxygen to fractional inspired oxygen $\left.\left(\mathrm{PaO}_{2} / \mathrm{FiO}_{2}\right)<300 \mathrm{~mm} \mathrm{Hg} ; 2\right)$ Noninvasive mechanical ventilation (bilevel positive airway pressure/continuous positive airway pressure (BiPAP/CPAP)) use; 3) Invasive mechanical ventilation; 4) Use of vasopressors; 5) Intensive care unit (ICU) admission; 6) Death.

\section{Data collection}

Data were extracted from the hospital's electronic medical record by two internal medicine resident physicians who were trained on the inclusion and exclusion criteria and use of a data extraction form to collect data by the principal investigator. Data extraction was performed in August 2021. Before and during data extraction, meetings of the research team and data extractors were convened to ensure uniformity of data extraction. A third internal medicine resident independently verified the data of eight randomly selected patient charts (approximately $10 \%$ of the cohort).

\section{Statistical analysis}

All the patients that fulfilled the inclusion criteria were included in the study; hence no sample size estimation was performed. The frequency and percentages of categorical variables were calculated. Mean values with standard deviations (SD) were calculated for continuous variables if normally distributed, and as median with interquartile range if not normally distributed. Differences in continuous variables between the groups were evaluated using independent-sample t-Test or nonparametric MannWhitney $U$ test, as appropriate. Differences in the categorical variables between the groups were analyzed using Chi-square or
Fisher's exact test. Univariate analysis was performed to explore the association of all variables to severe SARS-CoV-2 infection. Variables with a $\mathrm{P}$ value $<0.20$ were selected for multivariable logistic regression (LR) analysis (Model-1). The first LR model (Model-1) had high chances of overfitting due to large numbers of the predictor variables. Hence, the variance of inflation (VIF) was calculated to eliminate multicollinearity among the variables. Five variables with the lowest VIF scores were included (Model-2). The area under the receiver operating curve (AUC) was compared between the two models. The detailed information of LR models is available here (Supplementary Material 1-5, www.jocmr.org). Statistical analysis was performed using STATA 16.1 (Stata Corp, College Station, TX).

\section{Results}

\section{Primary outcome}

Seventy-eight patients were hospitalized with breakthrough SARS-CoV-2 infection, which represented 0.12\% $(78 / 67,223)$ of all vaccinated individuals within CFVHS.

\section{Secondary outcomes}

Twenty-five of $67,223(0.04 \%)$ fully or partially vaccinated individuals developed severe breakthrough SARS-CoV-2 infection. Symptoms started on mean 8 (SD: 6.60) and 6 (SD: 5.65) days post-vaccination in partially and fully vaccinated patients, and the mean length of hospital stay was 8 (SD: 7.50) and 6 (SD: 5.91) days, respectively. Only 8 of $78(10.2 \%)$ patients had symptom onset greater than 2 weeks after their last vaccine dose. There were five $(13 \%)$ and eight $(20 \%)$ deaths in partially and fully vaccinated patients, respectively. Patients who were either partially or fully vaccinated and had breakthrough infections had a mean age of 70 (SD: 11.35) and 73 (SD: 14.14) years, respectively, and were predominantly White. The male constituted $48 \%$ of partially vaccinated patients and $59 \%$ of fully vaccinated patients. The distribution of hospitalization of breakthrough infections formed two incidence peaks: one in January-February (40\%) and another in July-August (34\%) (Table 1).

Multivariable LR analysis was performed to explore variables that were independently associated with the severe breakthrough SARS-CoV-2 infection. Male patients had 4.03 times the odds of developing severe breakthrough SARS-CoV-2 infection than female patients (aOR: 4.03, 95\% confidence interval (CI): 1.21 - 13.40), and the immunocompromised status had 6.32 times the odds of developing severe breakthrough SARSCoV-2 infection (aOR: 6.32, 95\% CI: 1.48 - 26.18). Similarly, each $1 \%$ increase in oxygen saturation $\left(\mathrm{SPO}_{2}\right)$ decreased the odds of developing severe breakthrough SARS-CoV-2 infection by $20 \%$ (aOR: $0.80,95 \%$ CI: $0.68-0.94$ ) (Table 2 ).

\section{Discussion}

In this single health system retrospective study, we found that 
Table 2. Logistic Regression (LR) Model-2 Exploring Factors Associated With Severe Breakthrough SARS-CoV-2 Infection

\begin{tabular}{llll} 
& aOR & 95\% CI & P value \\
\hline Fully vaccination status (vs. partially) & 2.42 & $0.71-8.28$ & 0.15 \\
Sex (male) & 4.03 & $1.21-13.40$ & 0.023 \\
$\mathrm{SPO}_{2}(\%)$ & 0.80 & $0.68-0.94$ & 0.009 \\
$\mathrm{COPD}$ & 0.24 & $0.05-27.01$ & 0.063 \\
Immunocompromised status & 6.32 & $1.48-26.18$ & 0.013 \\
\hline
\end{tabular}

Area under the receiver operating curve (AUC) for the LR model $0.813,95 \% \mathrm{Cl}: 0.71-0.91$ (LR model controlled for: fully vs. partially vaccinated status, sex, $\mathrm{SPO}_{2}, \mathrm{COPD}$ and immunocompromised status). aOR: adjusted odds ratio; $\mathrm{Cl}$ : confidence interval; $\mathrm{SPO}_{2}$ : oxygen saturation; $\mathrm{COPD}$ : chronic obstructive pulmonary disease.

breakthrough SARS-CoV-2 infection was rare $(0.12 \%)$. The development of severe breakthrough SARS-CoV-2 infection among the vaccinated was very rare $(0.04 \%)$. Multivariable LR analysis found no significant association of fully or partially vaccinated status in developing severe breakthrough SARSCoV-2 infection, but male sex and immunocompromising conditions were associated with the increased odds of developing severe breakthrough SARS-CoV-2 infection. As of October 18 , the CDC reported a total of 41,127 breakthrough infections $(0.021 \%)$ that needed hospitalization out of 189 million vaccinated individuals [19]. This low breakthrough SARS-CoV-2 infection was consistent in our study.

In this study, we based our definition of severe SARSCoV-2 infection on Pfizer-BioNTech and Moderna Inc.'s RCTs $[2,3]$. The ranges of clinical parameters used to define severe disease in those RCTs were broad, hence every patient who qualified for the study was found to fit the criteria for severe infection at some point during hospitalization. In an attempt to be more specific, we modified our definition of severe breakthrough SARS-CoV-2 infection, and according to that definition, 25 patients qualified for severe SARS-CoV-2 infection. Thirteen of 25 patients with severe breakthrough infections died and accounted for 17\% (13/78) of patients hospitalized with breakthrough SARS-CoV-2 infection. This mortality rate was high and consistent with CDC reporting as of October 18, $2021(26 \%, 10,857 / 41,127)$. However, clinical details of the patients who died in this CDC report were not available. Upon chart review, we found that 12 of the 13 patients were made do-not-resuscitate (DNR) before death. In addition, these patients had multiple comorbidities at baseline and had frequent hospitalizations. This may have contributed to the higher mortality rate seen in our study. The remaining patient who died and was not DNR before death was a young patient who died of postoperative massive saddle pulmonary embolism. The details of 13 patients who had mortality are outlined here (Supplementary Materials 6, www.jocmr.org).

Emerging evidence supports the concept that patients on chronic immunosuppression or on treatment for malignancy may not mount sufficient immune responses to mRNA vaccine, which may predispose them to breakthrough infections. For example, a recent study suggested that CID patients who received both doses of mRNA vaccine had sufficient antispike immunoglobulin $\mathrm{G}(\mathrm{IgG})$ antibody titers, but the titers were suboptimal if they were receiving chronic corticosteroid or B-cell depletion chemotherapy [20]. Similarly, in SOT recipients, the prevalence of anti-SARS-CoV-2 antibodies was $34 \% 1$ month after the second dose [21]. Our study considered patients immunocompromised if they were receiving chronic corticosteroid therapy or immunotherapy for CID, chemotherapy for malignancy, SOT recipients, or AIDS patients. In this study, we found that immunocompromised status was associated with the increased odds of developing severe COVID-19 breakthrough disease.

In our study, we noted two spikes of breakthrough SARSCoV-2 infection. The first spike was in January-February, and the second spike was seen in July-August, 2021. Only 10\% were found to have onset of symptoms 2 weeks or later postvaccination (Table 1). The second spike is probably due to the delta variant, which is currently the predominant variant in the USA, and mRNA vaccines are less effective against the delta variant [22], and relaxed public health rules such as lifting mask mandates which occurred in North Carolina [23]. Our knowledge of waning immunity from these vaccines is evolving, but it is possible that due to decreasing titers of antiSARS-CoV-2 antibodies with time, the effectiveness of the mRNA vaccines has been decreasing.

Our study had a few limitations. First, our number of breakthrough infections could be an underestimate. If patients who were vaccinated in CFVHS were not admitted to CFVHS for symptomatic breakthrough infection, this could result in a falsely low number of breakthrough infections. Patients who were vaccinated outside CFVHS or self-reported to be vaccinated were excluded from the study due to lack of evidence of vaccination. Twelve percent population in North Carolina does not have access to health care and is less likely to seek medical attention [24]. Patients admitted for breakthrough SARS-CoV-2 infections, as well as total vaccinated individuals, might have been underrepresented. This might have caused selection bias in the study. CFVHS has a wide catchment area and had approximately 130,000 ED visits in 2020, the highest in North Carolina. This makes it less likely that many local patients would have sought care at other health networks for treatment. Also, it is reassuring that the breakthrough SARS-CoV-2 infection rate we found was similar to what CDC reported in nationwide data [19]. This further reassured that the selection bias was minimal. Second, the sample size was small. Since this study investigated a very specific finding we believe it is acceptable. Third, in the LR analysis, we found that each $1 \%$ increase in $\mathrm{SPO}_{2}$ was 
associated with decreased odds of severe SARS-CoV-2 infection development, but it was not controlled for the supplemental oxygen requirement. Fourth, this study did not have data on the variant of SARS-CoV-2 and anti-SARS-CoV-2 antibody titers, so it was not possible to determine if the second spike of hospitalizations was due to declining immunity or due to a new variant of the virus, or both. Lastly, because of the retrospective nature of the study, we could not determine the circumstantial reason for the partial vaccination status. Despite these limitations, we believe that this is the first study that described the clinical characteristics of breakthrough and severe breakthrough SARS-CoV-2 infections in the general population. A few recent studies on breakthrough SARS-CoV-2 infection were limited to health care workers, prison inmates, gold miners, and nursing home residents [7$10,12,17]$. In this study, we could not discuss about the viral vector COVID-19 vaccine as none of the patients included in the study received the Janssen Pharmaceuticals COVID-19 vaccine.

\section{Conclusions}

In this single health system study, we found that the breakthrough SARS-CoV-2 infections are rare. Male sex and immunocompromised status were associated with increased odds of developing the severe-COVID-19 breakthrough disease. This study supports that the vaccine continues to be an effective intervention to prevent severe SARS-CoV-2 infection and hospitalization. Providers and health systems must continue aggressive education on the importance of vaccination.

\section{Supplementary Material}

Suppl 1. Univariate analysis predicting severe breakthrough COVID-19 infection.

Suppl 2. Logistic regression model-1 exploring factors associated with severe breakthrough COVID-19 infection.

Suppl 3. Variance inflation factor (VIF) measurement of the variables in logistic regression model-1.

Suppl 4. Logistic Regression Model-2 exploring factors associated with severe breakthrough COVID-19 infection.

Suppl 5. Area under receiver operating curve of logistic regression models, model-1 and model-2.

Suppl 6. Clinical details of patients with severe breakthrough COVID-19 infection having mortality.

\section{Acknowledgments}

None to declare.

\section{Financial Disclosure}

None to declare.

\section{Conflict of Interest}

None to declare.

\section{Informed Consent}

The informed consent was waived by IRB of CFVHS (IRB ID: NR1085-21) as the study was a retrospective study.

\section{Author Contributions}

RA, SK, NK, BS, MG and AT contributed to manuscript writing, data analysis, project management, and approved the final manuscript for submission. RA conceptualized the study. AT supervised the study. RA assumes responsibility for the integrity of the work as a whole, from inception to published article.

\section{Data Availability}

The authors declare that data supporting the findings of this study are available within the article and the supplementary material.

\section{References}

1. The true death toll of COVID-19: estimating global excess mortality [Internet]. [cited Sep 1, 2021]. Available from: https://www.who.int/data/stories/the-true-death-toll-ofcovid-19-estimating-global-excess-mortality.

2. Polack FP, Thomas SJ, Kitchin N, Absalon J, Gurtman A, Lockhart S, Perez JL, et al. Safety and efficacy of the BNT162b2 mRNA COVID-19 vaccine. N Engl J Med. 2020;383(27):2603-2615.

3. Baden LR, El Sahly HM, Essink B, Kotloff K, Frey S, Novak R, Diemert D, et al. Efficacy and safety of the mRNA-1273 SARS-CoV-2 vaccine. N Engl J Med. 2021; 384(5):403-416.

4. Dagan N, Barda N, Kepten E, Miron O, Perchik S, Katz MA, Hernan MA, et al. BNT162b2 mRNA COVID-19 vaccine in a nationwide mass vaccination setting. N Engl J Med. 2021;384(15):1412-1423.

5. Haas EJ, Angulo FJ, McLaughlin JM, Anis E, Singer SR, Khan F, Brooks N, et al. Impact and effectiveness of mRNA BNT162b2 vaccine against SARS-CoV-2 infections and COVID-19 cases, hospitalisations, and deaths following a nationwide vaccination campaign in Israel: an observational study using national surveillance data. Lancet. 2021;397(10287):1819-1829.

6. Hacisuleyman E, Hale C, Saito Y, Blachere NE, Bergh M, Conlon EG, Schaefer-Babajew DJ, et al. Vaccine breakthrough infections with SARS-CoV-2 variants. N Engl J Med. 2021;384(23):2212-2218.

7. Farinholt T, Doddapaneni H, Qin X, Menon V, Meng Q, 
Metcalf G, Chao H, et al. Transmission event of SARSCoV-2 Delta variant reveals multiple vaccine breakthrough infections. medRxiv. 2021.

8. Brinkley-Rubinstein L, Peterson M, Martin R, Chan P, Berk J. Breakthrough SARS-CoV-2 Infections in Prison after Vaccination. N Engl J Med. 2021;385(11):1051-1052.

9. Jacobson KB, Pinsky BA, Montez Rath ME, Wang H, Miller JA, Skhiri M, Shepard J, et al. Post-vaccination SARS-CoV-2 infections and incidence of presumptive B.1.427/B.1.429 variant among healthcare personnel at a northern California academic medical center. Clin Infect Dis. 2021.

10. Teran RA, Walblay KA, Shane EL, Xydis S, Gretsch S, Gagner A, Samala U, et al. Postvaccination SARS-CoV-2 infections among skilled nursing facility residents and staff members - Chicago, Illinois, December 2020-March 2021. MMWR Morb Mortal Wkly Rep. 2021;70(17):632-638.

11. Pollett SD, Richard SA, Fries AC, Simons MP, Mende K, Lalani T, Lee T, et al. The SARS-CoV-2 mRNA vaccine breakthrough infection phenotype includes significant symptoms, live virus shedding, and viral genetic diversity. Clin Infect Dis. 2021:ciab543.

12. Vignier N, Berot V, Bonnave N, Peugny S, Ballet M, Jacoud E, Michaud C, et al. Breakthrough infections of SARS-CoV-2 gamma variant in fully vaccinated gold miners, French Guiana, 2021. Emerg Infect Dis. 2021; 27(10):2673-2676.

13. McEwen AE, Cohen S, Bryson-Cahn C, Liu C, Pergam SA, Lynch J, Schippers A, et al. Variants of concern are overrepresented among post-vaccination breakthrough infections of SARS-CoV-2 in Washington State. Clin Infect Dis. 2021:ciab581.

14. Mostafa HH, Luo CH, Morris CP, Li M, Swanson NJ, Amadi A, Gallagher N, et al. SARS-CoV-2 Infections in mRNA vaccinated individuals are biased for viruses encoding spike E484K and associated with reduced infectious virus loads that correlate with respiratory antiviral IgG levels. medRxiv. 2021.

15. Kustin T, Harel N, Finkel U, Perchik S, Harari S, Tahor M, Caspi I, et al. Evidence for increased breakthrough rates of
SARS-CoV-2 variants of concern in BNT162b2-mRNAvaccinated individuals. Nat Med. 2021;27(8):1379-1384.

16. Butt AA, Khan T, Yan P, Shaikh OS, Omer SB, Mayr F. Rate and risk factors for breakthrough SARS-CoV-2 infection after vaccination. J Infect. 2021;83(2):237-279.

17. Geysels D, Van Damme P, Verstrepen W, Bruynseels P, Janssens B, Smits P, Naesens R. SARS-CoV-2 vaccine breakthrough infections among healthcare workers in a large Belgian hospital network. Infect Control Hosp Epidemiol. 2021:1-2.

18. CDC COVID-19 Vaccine Breakthrough Case Investigations Team. COVID-19 Vaccine breakthrough infections reported to CDC - United States, January 1-April 30, 2021. MMWR Morb Mortal Wkly Rep. 2021;70(21):792-793.

19. COVID-19 Breakthrough Case Investigations and Reporting | CDC [Internet]. 2021 [cited Sep 2, 2021]. Available from: https://www.cdc.gov/vaccines/covid-19/health-departments/breakthrough-cases.html.

20. Deepak P, Kim W, Paley MA, Yang M, Carvidi AB, Demissie EG, El-Qunni AA, et al. Effect of immunosuppression on the immunogenicity of mRNA vaccines to SARS-CoV-2: a prospective cohort study. Ann Intern Med. 2021;174(11):1572-1585.

21. Marion O, Del Bello A, Abravanel F, Couat C, Faguer S, Esposito L, Hebral AL, et al. Safety and immunogenicity of anti-SARS-CoV-2 messenger RNA vaccines in recipients of solid organ transplants. Ann Intern Med. 2021; 174(9):1336-1338.

22. Lopez Bernal J, Andrews N, Gower C, Gallagher E, Simmons R, Thelwall S, Stowe J, et al. Effectiveness of COVID-19 Vaccines against the B.1.617.2 (Delta) Variant. N Engl J Med. 2021;385(7):585-594.

23. CDC. Coronavirus Disease 2019 (COVID-19) [Internet]. Centers for Disease Control and Prevention. 2020 [cited Sep 1, 2021]. Available from: https://www.cdc.gov/ coronavirus/2019-ncov/variants/delta-variant.html.

24. Cumberland County CHNA Report Final.pdf [Internet]. [cited Nov 25, 2021]. Available from: https://schs.dph. ncdhhs.gov/units/ldas/cha2018/Cumberland_County\%20 CHNA\%20Report\%20Final.pdf. 